\title{
Therapeutic landscape of metastatic non-small-cell lung cancer in Canada in 2020
}

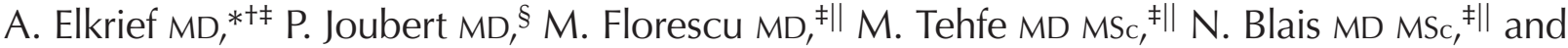 \\ B. Routy $M D P h D^{\ddagger \|}$
}

\begin{abstract}
Lung cancer is the most commonly diagnosed cancer in Canada and remains associated with high mortality. Nevertheless, recent advances in the fields of immuno-oncology and precision medicine have led to significant improvements in clinical outcome in metastatic non-small-cell lung cancer (NSCLC). Those improvements were facilitated by a greater understanding of the biologic classification of NSCLC, which catalyzed discoveries of novel therapies. Here, we present a comprehensive review of the recent avalanche of practice-changing trials in metastatic NSCLC, and we offer an approach to the management of this disease from a Canadian perspective. We begin with an overview of the pathologic and molecular characterization of metastatic NSCLC. Next, we review the indications for currently approved immune checkpoint inhibitors, and we provide an approach to the management of disease with a driver mutation. Finally, we address future avenues in both diagnostics and therapeutics for patients with advanced and metastatic NSCLC.
\end{abstract}

Key Words Lung cancer, immunotherapy, targeted therapy, chemotherapy, biomarkers

Curr Oncol. 2020 February;27(1):52-60

www.current-oncology.com

\section{INTRODUCTION}

For the 1 in 2 Canadians who will develop a malignancy during their lifetime, lung cancer remains the most common and the most deadly: each day, 58 Canadians die from lung cancer ${ }^{1}$. Despite the implementation of tobacco cessation programs and some access to screening programs in high-risk populations ${ }^{2}$, more than half the patients with non-small-cell lung cancer (NSCLC) are still diagnosed with metastatic disease ${ }^{3}$. Since about 2010, unprecedented breakthroughs have occurred in the treatment of advanced NSCLC, in part because of the emergence of immune checkpoint inhibition (ICI) and targeted therapy, which provide long-term control in some patients. Indeed, the therapeutic landscape of metastatic NSCLC is becoming increasingly complex, in part because of the use of various biomarkers in many clinical trials and because of new toxicity profiles. Sound knowledge and understanding of that landscape are therefore required for all practitioners involved in the care of the patient with metastatic NSCLC. In this review, we begin with the pathology evaluation of the disease leading to biomarker-driven therapy, followed by a comprehensive evidenced-based approach to current management of advanced or metastatic NSCLC in Canada in 2020. Finally, we provide a commentary about potential future options for diagnostics and therapeutics that are yet to be approved in Canada.

\section{REVIEW}

\section{What Are the Pathology Characterizations of NSCLC and Why Are They Important?}

Histologic and Molecular Characterizations of NSCLC Advanced NSCLC includes patients with either stage IV or stage III disease not eligible for locoregional treatment modalities (according to the 8th version of the TNM classification $)^{4}$. The treatment of such patients relies on systemic therapy tailored to pathologic, histologic, and molecular subtype. A standard pathology evaluation to determine

This series is brought to you in partnership with the Canadian Association of General Practitioners in Oncology.

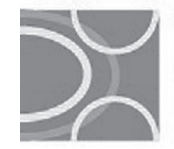

\section{THE CANADIAN ASSOCIATION OF GENERAL PRACTITIONERS IN ONCOLOGY}


histology (adenocarcinoma vs. squamous cell carcinoma vs. other more rare variants) is therefore essential. Defining the histology guides the molecular testing and choice of therapy. For example, pemetrexed, when combined with cisplatin, is associated with improved overall survival (os) in patients with nonsquamous adenocarcinoma cell histology, and worse os when used in patients with squamous histology ${ }^{5}$.

Testing for molecular alterations in lung adenocarcinomas amenable to targeted therapy is now considered the standard of care $^{6-8}$. However, the number of genes approved to be tested according to clinical practice guidelines is not yet homogenous across Canadian institutions, and for rarer actionable mutations, reimbursement of highly active medications is not guaranteed ${ }^{8}$. Patients at higher risk for detection of those molecular alterations include those who have little or no prior smoking history, patients of female sex, younger patients, and patients of Asian descent ${ }^{9}$. Accordingly, based on an expert panel consensus opinion ${ }^{10}$, the current recommendation is also to test all tumours with an adenocarcinoma component, non-small-cell carcinoma not otherwise specified, sarcomatoid carcinoma, and squamous cell carcinomas with atypical clinical presentation such as young age ( $<50$ years), nonsmoking or mild smoking habit ( $<10$ pack-years), or presence of a peripheral lesion. National clinical practice consensus recommends that, for those patients, EGFR gene mutations, ALKgene fusions, $R O S 1$ rearrangements, and $B R A F \mathrm{~V} 600 \mathrm{E}$ mutations be tested $^{8,11,12}$. NTRK gene fusions, ERBB2 mutations or HER2 amplifications, MET exon 14 skipping mutations of whole-gene amplifications, RET translocations, and RAS mutations are emerging alterations with novel applications in the treatment of NSCLC, although funding and regulatory issues preclude widespread diagnostic testing in Canada at this time ${ }^{8}$. Lastly, it is also recommended that, for all patients with advanced tumours, PD-L1 status be tested (according to "fit-for-purpose" principles ${ }^{13}$ ) using the Dako 22C3 pharmDx assay on a Dako Autostainer (Dako Corporation, Glostrup, Denmark ${ }^{11}$, because the results will affect the choice and sequence of immunotherapy and chemotherapy, as we outline in detail later in this article. Notably, other PD-L1 companion testing such as 28-8, SP-142, SP-263, and 73-10 is not currently reimbursed or recommended.

\section{Current Management of NSCLC in the First Line}

\section{Introduction of ICI in NSCLC}

Landmark trials CheckMate 017 and 057 compared nivolumab with docetaxel in previously treated metastatic NSCLC and demonstrated superior os in the nivolumab group, forever changing the treatment algorithm in both the squamous and nonsquamous histologies. Unlike previous findings in historical chemotherapy trials, ICI provided a sustained response in $20 \%$ of patients at 4 years ${ }^{14}$. That observation led to the study of ICI in first-line settings, with unprecedented improvements in patient outcomes. As a result, ICI is now a cornerstone in the first-line setting for all eligible patients without a driver mutation, and the choice of regimen depends on PD-L1 status (Figure 1).

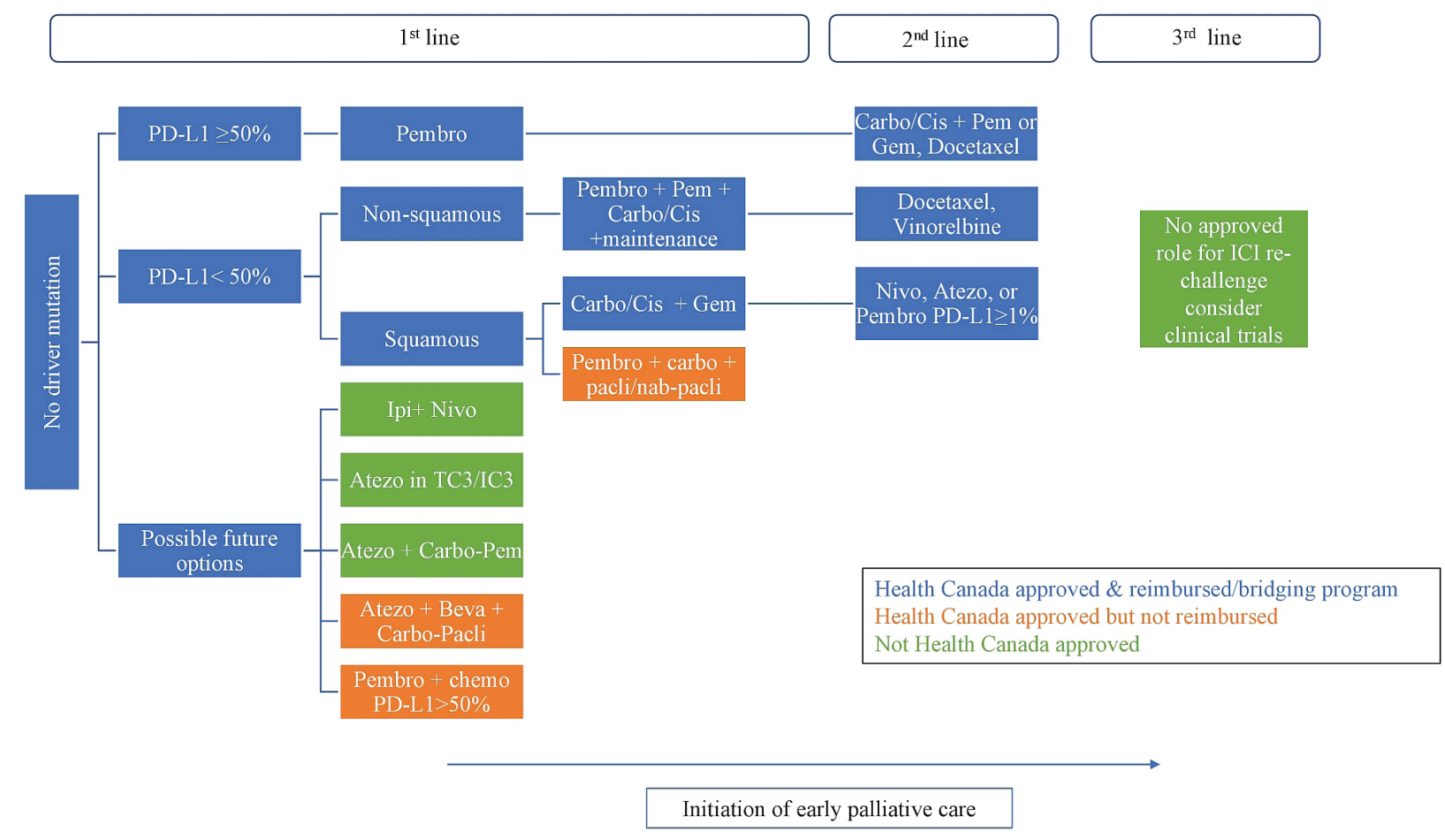

FIGURE 1 Immune checkpoint inhibition in the management of metastatic non-small-cell lung cancer without a driver mutation in January 2020. Pembro = pembrolizumab; Carbo = carboplatin; Cis = cisplatin; Gem = gemcitabine; Pem = pemetrexed; Nivo = nivolumab; Atezo = atezolizumab; $\mathrm{ICI}=$ immune checkpoint inhibition; Pacli = paclitaxel; Ipi = ipilimumab; Beva = bevacizumab. 


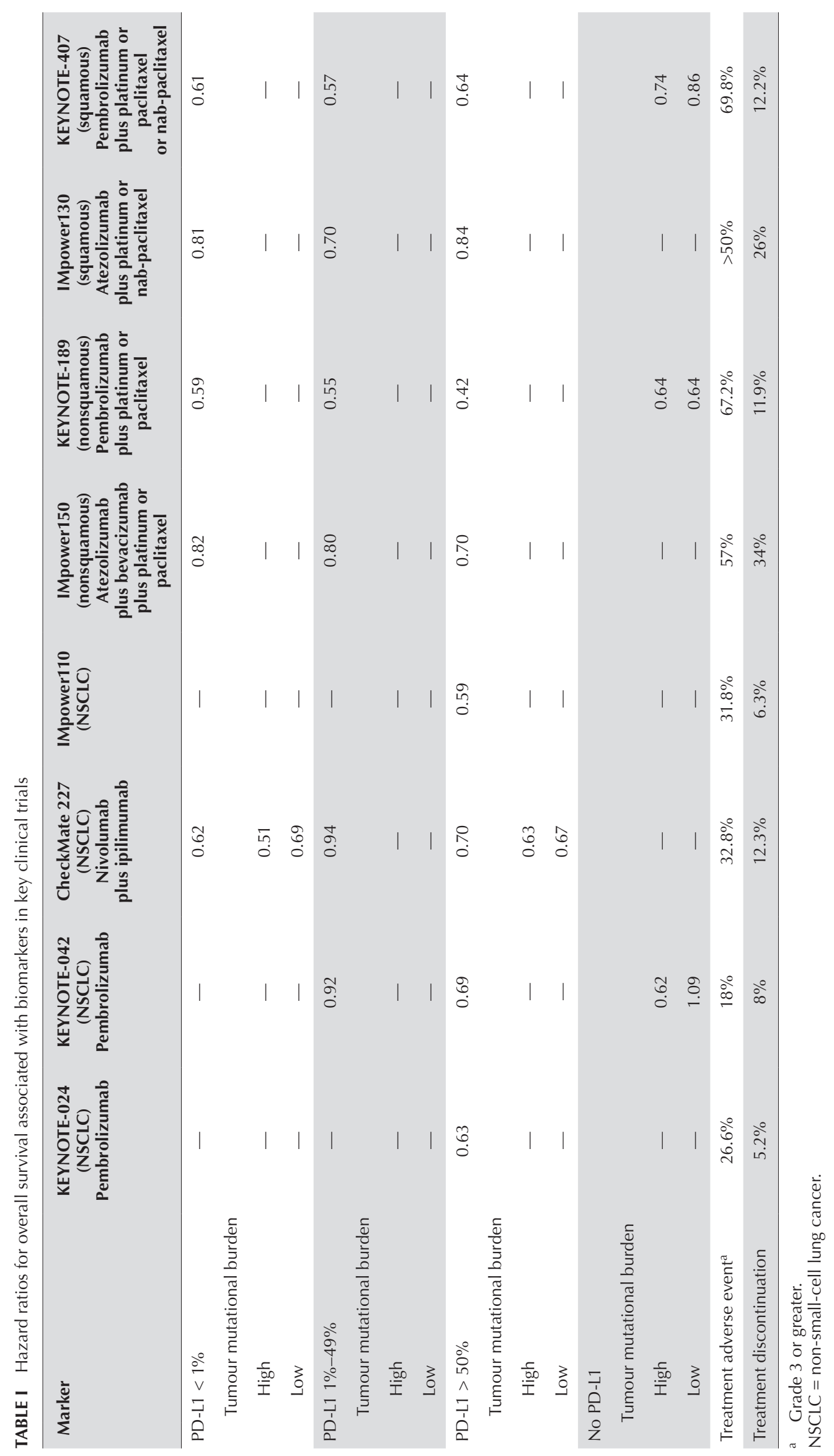




\section{Anti-PD-1 and PD-L1 Monotherapy}

Single-agent pembrolizumab is indicated in patients with previously untreated advanced NSCLC with a PD-L1 status of $50 \%$ or greater. In the phase III KEYNOTE- 024 randomized clinical trial, 305 patients with previously untreated NSCLC having no driver mutation were randomized to receive physician's choice of platinum-based chemotherapy or single-agent pembrolizumab ${ }^{15}$. Patients treated with single-agent pembrolizumab experienced significantly longer Os [26.6 months vs. 14.2 months; hazard ratio (HR): $0.63 ; 95 \%$ confidence interval (CI): 0.47 to $0.86 ; p=0.002$ ], and more than $40 \%$ of patients in the pembrolizumab arm were still alive at 3 years (Table I). Patients in the pembrolizumab arm also experienced less-frequent grade III or greater adverse events. Typically, treatment is discontinued after 2 years or at progression, or if serious immune-related adverse events occur.

The greater efficacy of pembrolizumab in patients with a PD-L1 status of $50 \%$ or greater was corroborated by KEYNOTE-042. Although pembrolizumab proved to be less toxic than standard chemotherapy in that trial, it was not superior to chemotherapy in patients with PD-L1 expression in the $1 \%-49 \%$ range $^{16}$. Conversely, single-agent nivolumab was evaluated in previously untreated patients with a PD-L1 status greater than 5\% in the CheckMate 026 trial, where it failed to show superiority ${ }^{17}$. In the same vein, the MYSTIC trial failed to show benefit for combination durvalumabtremelimumab compared with standard chemotherapy ${ }^{18}$.

\section{Nonsquamous NSCLC: Anti-PD-1 or PD-L1 in Combination with Chemotherapy}

A combination of cytotoxic chemotherapy and pembrolizumab is indicated in previously untreated patients with advanced nonsquamous NSCLC regardless of PD-L1 status and is broadly preferred in patients with less than $50 \%$ PD-L1 expression. In the phase III KEYNOTE-189 clinical trial, 616 patients were randomized to receive platinum-based chemotherapy (carboplatin or cisplatin) with pemetrexed, plus either pembrolizumab or placebo for up to 35 cycles, plus pemetrexed maintenance therapy ${ }^{19}$. The combination regimen was associated with an improved progression-free survival (PFS) of 8.8 months compared with 4.9 months (HR: 0.52 ; $95 \%$ CI: 0.43 to $0.64 ; p<0.001$ ) and an improved os rate (Kaplan-Meier probabilities for proportion of patients alive at 12 months: $69 \%$ vs. $49 \%$; HR: $0.49 ; 95 \%$ CI: 0.38 to $0.64 ; p<0.001$; Table I). Similarly, preliminary data from the IMpower132 trial demonstrated a PFs benefit (median: 7.6 months vs. 5.2 months) with the addition of atezolizumab to pemetrexed-based chemotherapy ${ }^{20}$. The interim analysis suggested an improvement in os of 4.7 months with the addition of atezolizumab.

Although cisplatin-based regimens are considered slightly more effective than carboplatin and nonplatinum-based regimens, the advantage of cisplatin over carboplatin remains an unanswered question with respect to the chemotherapy-ICI combination ${ }^{19}$.

\section{Squamous NSCLC with Less Than 50\% PD-L1}

In patients with metastatic NSCLC and squamous histology, the KEYNOTE-407 registration trial also assessed the role of ICI in squamous histology and demonstrated that the addition of pembrolizumab to chemotherapy with carboplatin plus paclitaxel or nab-paclitaxel was associated with improved PFS and os in patients with previously untreated metastatic disease. Similarly, the IMpower131 study randomized patients into 3 arms (using combinations not currently approved in Canada):

Arm A: atezolizumab plus carboplatin-paclitaxel

Arm B: atezolizumab plus carboplatin-nab-paclitaxel Arm C: chemotherapy alone (carboplatin-nab-paclitaxel)

The investigators found a benefit for arm B compared with arm C (6.3 months vs. 5.6 months; HR: 0.716; 95\% CI: 0.603 to $0.848 ; p=0.0001)^{21}$. Interestingly, those findings were independent of PD-L1 status ${ }^{22}$ (Table I).

\section{Patterns of Response in NSCLC}

\section{What Are the New Patterns of Response in Metastatic NSCLC?}

To address the various ways in which tumours respond to ICI compared with standard chemotherapy, the immune Response Evaluation Criteria in Solid Tumors were implemented during the study of immunotherapy agents ${ }^{23}$. When using ICI, several unusual responses can be observed, such as durable response (no standard definition currently exists), pseudo-progression (rare, describes regression of tumour index lesions after initial progression), hyperprogression $\left(5 \%-10 \%\right.$ of patients in some studies ${ }^{24,25}$, with heterogeneous definitions; connotes rapidly progressive disease), and dissociated response (some tumours grow and others regress in a given individual) ${ }^{23}$.

\section{Treatment of Older Adults}

\section{How Are Special Populations Treated?}

The treatment of older adults with standard cytotoxic chemotherapeutic agents is associated with increased toxicity and worse outcomes ${ }^{26,27}$, a situation that might not be the case with immunotherapy. With immunotherapy, performance status seems to be a more accurate predictor of response, and older adults have outcomes similar to those seen in their younger counterparts. That observation also seems to extend to the overall safety and tolerability of the agents ${ }^{28,29}$.

Current Management of NSCLC in the Second Line Cytotoxic chemotherapy is the mainstay for patients who have progressed on standard first-line treatment (aside from patients with targetable mutations who have other options). Regimens are decided as a function of prior therapies, performance status, comorbidities, and organ function. A platinum doublet is typically used after progression on pembrolizumab monotherapy, or docetaxel for patients who have already been exposed to a platinum doublet with pembrolizumab in the first line. Other single-agent regimens such as gemcitabine or vinorelbine can also be used in third- and fourth-line settings ${ }^{30,31}$. For patients who did not receive ICI in the first-line setting, nivolumab (CheckMate 017 and CheckMate 057), pembrolizumab 
(KEYNOTE-010), or atezolizumab (OAK) can be used after progression on chemotherapy ${ }^{32-34}$.

\section{Current Management of Patients with a Driver Mutation}

\section{EGFR}

Worldwide, about $20 \%$ of all patients with advanced NSCLC are found to have a somatic activating EGFR mutation, with the 2 most common mutations being EGFR L858R and EGFR exon 19 deletion ${ }^{35}$ (Figure 2). Such mutations are more common in patients of Asian ethnicity or nonsmoking status. In several large randomized clinical trials (LUX-Lung 3 for afatinib, WJTOG3405 for gefitinib, and EURTAC for erlotinib), EGFR inhibition with first-generation and second-generation tyrosine kinase inhibitors (TKIs), compared with standard cytotoxic chemotherapy, was associated with improved $\mathrm{PFS}^{36-38}$. In the FLAURA trial, osimertinib, a third-generation TKI, was compared with first-generation TKI and was associated with improved os and fewer severe adverse events in 556 patients with previously untreated EGFR-positive disease. The frequency of disease progression in the central nervous system was also lower in the osimertinib group ${ }^{39}$.

Osimertinib, erlotinib, gefitinib, and afatinib are all approved as first-line therapy for patients with EGFRpositive metastatic NSCLC in Canada. Osimertinib is approved for second-line treatment in patients with EGFR-positive disease progressing on first- and second-generation TKIs and having de novo T790M mutation (AURA3) ${ }^{40}$. The most common side effects seen with EGFR TKIs are rash, diarrhea, and cytopenias (lymphopenia, thrombocytopenia).

Over the past few years, the use of liquid biopsy to assess T790M status in patients progressing on an EGFR
TKI has also been implemented in some Canadian centres and is under evaluation for reimbursement in several provinces. The technique, which requires a single blood sample, presents notable advantages over invasive sampling. However, given test sensitivity varying from $60 \%-80 \%$, a negative liquid biopsy result is currently recommended to be complemented with evaluation of a tissue or a cytology specimen, in particular if the native activating mutation is not found ${ }^{41}$.

\section{ALK}

$A L K$ rearrangements are found in fewer than $5 \%$ of patients with metastatic NSCLC. Nevertheless, the therapeutic implications of targeting this driver mutation are important, given that an improved os is associated with crizotinib (a first-generation TKI targeting ALK) compared with standard chemotherapy ${ }^{42}$. The next-generation ALK TKIs brigatinib (ALTA-1L) ${ }^{43}$ and alectinib (ALEX) ${ }^{44}$ have now been shown to be superior to crizotinib. At the time of writing, access to those standard-of-care therapies was variable in Canada. The most important side effect for both brigatinib and alectinib is increased liver enzymes, and brigatinib is also associated with a particular manifestation of pneumonitis (early-onset pulmonary event) and bradycardia.

\section{Rarer Driver Mutations in NSCLC}

Targeting of rarer mutations such as ROS1 with crizotinib, $N T R K$ with larotrectinib, RET with selpercatinib, and $B R A F$ V600E with dabrafenib-trametinib are emerging therapeutic options. If a patient has a rare driver mutation, a second opinion at an academic centre is suggested, as is consideration for a clinical trial.

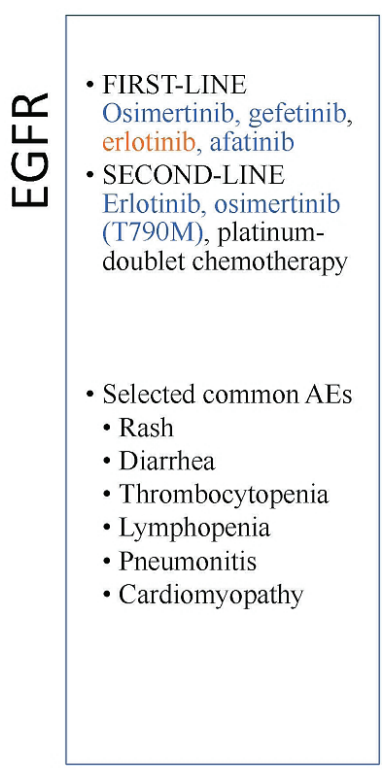

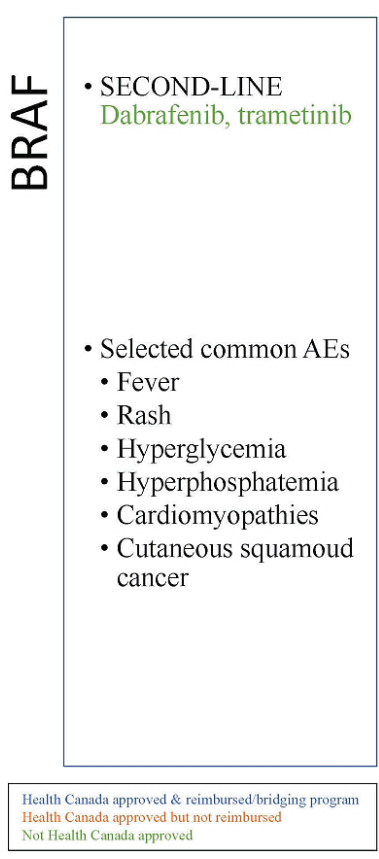

FIGURE 2 Summary of selected targeted agents for the management of metastatic non-small-cell lung cancer with a driver mutation in January 2020. $\mathrm{AEs}=$ adverse events; $\mathrm{ALT}=$ alanine transaminase; $\mathrm{AST}=$ aspartate transaminase; $\mathrm{CK}=$ creatine kinase; $\mathrm{ICl}=$ immune checkpoint inhibition . 


\section{Role of Immunotherapy in Patients with Driver Mutations}

A large retrospective study of real-world data for 551 patients with driver mutation-positive disease evaluated the role of ICI in that population. Use of ICI induced a response in a small proportion of patients, with patients having RET mutations and $A L K$ rearrangements experiencing little to no response. Patients with driver mutation-positive disease should therefore receive standard targeted therapy followed by chemotherapy in the second-line setting ${ }^{45}$. Thus far, the IMpower150 trial is the only study showing a suggestion of clinical activity with ICI (atezolizumab) in a subgroup analysis of patients with EGFR-positive disease treated after failure of standard TKIs (when combined with bevacizumab and chemotherapy) ${ }^{46}$. It is important to note that most of the first-line clinical trials excluded participants with driver mutations.

\section{The Importance of Early Palliative Care}

Despite the advances already described, metastatic NSCLC is associated with significant morbidity, and most patients will progress on standard treatment. A randomized clinical trial of 151 patients with metastatic NSCLC showed that incorporation of early palliative care with standard oncologic care not only improved quality of life, but also os ${ }^{47}$. More recently, in a large retrospective cohort of more than 20,000 patients, those with advanced lung cancer who received palliative care within 30-365 days of diagnosis were found to experience improved survival ${ }^{48}$. It is therefore important to integrate palliative approaches early in the management of advanced NSCLC.

\section{Biomarkers of Therapeutic Success}

\section{What Are the Biomarkers of Therapeutic Success for Patients Receiving Immunotherapy and What Are the Unanswered Questions After Recent Publications and Presentations at the 2019 Meeting of the European Society for Medical Oncology?}

Despite unprecedented-nearly doubled-long-term os with ICI in metastatic NSCLC, many patients develop resistance to ICI agents. Moreover, the toxicities of those agents, known as immune-related adverse events, can lead to longterm morbidity. (Management of such events is beyond the scope of this review; however, excellent guidelines to aid clinicians in the management of immune-related adverse events are available ${ }^{49,50}$.) Given those important toxicity profiles, there is an urgent need to find biomarkers for therapeutic success. In a recent multi-omics prediction of the response to PD-1 and PD-L1 inhibitors, CD8+ T cell abundance, tumour mutational burden (TMB), and PD-1 expression were present in $80 \%$ of patients who responded to therapy ${ }^{51,52}$.

Many unanswered questions about interpretation of biomarkers remain, given the multitude of recent studies presented at the 2019 meeting of the European Society for Medical Oncology (Table I).

PD-L1 50\% or Greater Group: In the PD-L1 50\% or greater group, efficacies and toxicities are similar with the use of either pembrolizumab or atezolizumab monotherapy. However, the TC3/IC3 method using the underperforming SP162 antibody is required for testing PD-L1 status in patients receiving atezolizumab, and atezolizumab is not yet available for widespread use in Canada. Again, in the PD-L1 $50 \%$ or greater subgroup, combination ipilimumab-nivolumab (part of CheckMate 227) ${ }^{53}$ demonstrated acceptable efficacy (HR: $0.79 ; 95 \%$ CI: 0.65 to 0.96). However, the toxicity associated with ipilimumabnivolumab compared with anti-PD-1 or PD-L1 monotherapy makes this option less attractive in the absence of a signal for stronger clinical efficacy.

Combining pembrolizumab with chemotherapy in patients with PD-L1 $50 \%$ or greater remains a conundrum, especially given the latest evidence that patients with a low тмв do not respond to pembrolizumab alone, but they do respond to pembrolizumab plus chemotherapy. Indeed, pembrolizumab plus chemotherapy is efficacious regardless of TMB status. In contrast, a subgroup analysis from the MYSTIC trial showed that TMB was not a significant biomarker in the chemotherapy arm compared with the durvalumab arm. In the chemotherapy arm compared with the durvalumab-tremelimumab arm, no correlation between PD-L1 and TMB was observed, but TMB measured in blood was found to be a significant biomarker, validating the studies already described ${ }^{54}$.

To add another layer of complexity, the first published CheckMate 227 paper suggested that only patients with a high тмв (10 or more mutations per megabase) responded to ipilimumab-nivolumab. However, the latest results from that group showed that TMB was not actually predictive of response. Despite TMB serving as an important prognostic factor, its widespread use in Canada remains controversial and requires further validation in prospective trials given its high cost and a lack of standardization in technique and cut-off values.

PD-L1 1\%-49\% Group: In the PD-L1 1\%-49\% group (and keeping in mind that the treatments were never compared head-to-head), pembrolizumab plus chemotherapy seems to be the best option (HR: 0.55 ; $95 \%$ CI: 0.34 to 0.90 ); combination nivolumab-ipilimumab does not seem to be as efficacious (HR: 0.94; 95\% CI: 0.75 to 1.18). In addition, several options combining atezolizumab with chemotherapy represent interesting new alternatives.

Finally, in the PD-L1 less than 1\% group, chemotherapy plus pembrolizumab is the preferred option in Canada. Recent results from CheckMate 227 showing efficacy in terms of an os similar to that found with chemotherapy plus pembrolizumab might challenge that standard. Although the toxicities in the two groups were never compared headto-head, a higher incidence of grade 3 or greater events was observed in the chemotherapy plus pembrolizumab group.

\section{Other Biomarkers}

Lastly, the unforeseen role of the gut microbiome is also emerging as a major predictor of immunotherapy response ${ }^{55}$. Indeed, a recent systematic review demonstrated the independent negative predictive effect of antibiotics in patients receiving immunotherapy ${ }^{56,57}$, and novel clinical trials are under way to advantageously modify the 
gut microbiome so as to improve the response to immunotherapy. Finally, other concomitant medications-such as baseline corticosteroids at doses greater than $10 \mathrm{mg}$ daily-have been associated with negative outcomes in patients with metastatic NSCLC ${ }^{58}$.

\section{Future Directions}

\section{What Are the Future Directions in the Management of Metastatic NSCLC?}

Overall, the therapeutic landscape of metastatic lung cancer has been revolutionized in recent years. Nevertheless, further advances are needed to overcome resistance in both ICI and targeted therapy, and much work is currently dedicated to that concern. Moreover, the discovery of new therapeutic targets ${ }^{59}$ through next-generation sequencing (high throughput) are under way. Finally, advances in liquid biopsy analysis of circulating biomarkers in peripheral blood ${ }^{60}$, such as circulating tumour cells and circulating tumour DNA are paving the way to more personalized approaches.

\section{SUMMARY}

Advances in the molecular biology and genomics of lung cancer have drastically improved patient outcomes. In the current era, patients are treated using a multidisciplinary approach, receiving either immunotherapy, immunotherapy and chemotherapy combinations, or targeted therapy depending on their PD-L1, EGFR, ALK, ROS1, BRAF, and $N T R K$ statuses. Continued research innovations in the field of lung cancer are eagerly awaited.

\section{Key Points}

Treatment of advanced or metastatic NSCLC is becoming increasingly complex because of a recent avalanche of clinical studies involving ICI, ICI and chemotherapy combinations, and targeted therapy

It is essential that adequate histologic and molecular evaluation be performed for patients with metastatic NSCLC, specifically looking for PD-L1 expression and driver mutation status.

The correct interpretation of biomarkers of success in clinical trials is essential when choosing therapies for patients with metastatic NSCLC.

\section{ACKNOWLEDGMENTS}

The authors thank Dr. Sébastien Préville Ratelle and generous support from the Valeda and Charles-Émile Préfontaine family.

\section{CONFLICT OF INTEREST DISCLOSURES}

We have read and understood Current Oncology's policy on disclosing conflicts of interest, and we declare that we have none.

\section{AUTHOR AFFILIATIONS}

*Segal Cancer Centre, Sir Mortimer B. Davis Jewish General Hospital, Montreal; ${ }^{\dagger}$ Cedars Cancer Centre, McGill University Health Centre, Montreal; ${ }^{\ddagger}$ Centre de recherche du Centre hospitalier de l'Universtité de Montréal, Montreal; §Institut universitaire de cardiologie et de pneumologie de Québec, Quebec City; and "Division of Hematology-Oncology, Centre hospitalier de l'Université de Montréal, Montreal, QC.

\section{REFERENCES}

1. Canadian Cancer Statistics Advisory Committee. Canadian Cancer Statistics 2019. Toronto, ON: Canadian Cancer Society; 2019.

2. Canadian Task Force on Preventive Health Care. Recommendations on screening for lung cancer. CMAJ 2016;188:425-32.

3. Chen VW, Ruiz BA, Hsieh MC, Wu XC, Ries LA, Lewis DR. Analysis of stage and clinical/prognostic factors for lung cancer from SEER registries: AJCC staging and collaborative stage data collection system. Cancer 2014;120 (suppl 23):3781-92.

4. DetterbeckFC, Boffa DJ, Kim AW, Tanoue LT. The eighth edition lung cancer stage classification. Chest 2017;151:193-203.

5. Scagliotti GV, Parikh P, von Pawel J, et al. Phase III study comparing cisplatin plus gemcitabine with cisplatin plus pemetrexed in chemotherapy-naive patients with advanced-stage non-small-cell lung cancer. J Clin Oncol 2008;26:3543-51.

6. Ellis PM, Blais N, Soulieres D, et al. A systematic review and Canadian consensus recommendations on the use of biomarkers in the treatment of non-small cell lung cancer. J Thorac Oncol 2011;6:1379-91.

7. Melosky B, Cheema P, Agulnik J, et al. Canadian perspectives: update on inhibition of $A L K$-positive tumours in advanced non-small-cell lung cancer. Curr Oncol 2018;25:317-328.

8. Melosky B, Blais N, Cheema P, et al. Standardizing biomarker testing for Canadian patients with advanced lung cancer. Curr Oncol 2018;25:73-82.

9. Smolle E, Pichler M. Non-smoking-associated lung cancer: a distinct entity in terms of tumor biology, patient characteristics and impact of hereditary cancer predisposition. Cancers (Basel) 2019;11:E204.

10. Lindeman NI, Cagle PT, Aisner DL, et al. Updated molecular testing guideline for the selection of lung cancer patients for treatment with targeted tyrosine kinase inhibitors: guideline from the College of American Pathologists, the International Association for the Study of Lung Cancer, and the Association for Molecular Pathology. J Thorac Oncol 2018;13:323-58.

11. Planchard D, Popat S, Kerr K, et al. on behalf of the ESMO Guidelines Committee. Metastatic non-small cell lung cancer: ESMO clinical practice guidelines for diagnosis, treatment and follow-up. Ann Oncol 2018;29:iv192-237.

12. Friedlaender A, Banna G, Malapelle U, Pisapia P, Addeo A. Next generation sequencing and genetic alterations in squamous cell lung carcinoma: where are we today? Front Oncol 2019;9:166.

13. Cheung CC, Lim HJ, Garratt J, Won J, Gilks CB, Torlakovic EE. Diagnostic accuracy in fit-for-purpose PD-L1 testing. Appl Immunohistochem Mol Morphol 2019;27:251-7.

14. Antonia SJ, Borghaei H, Ramalingam SS, et al. Four-year survival with nivolumab in patients with previously treated advanced non-small-cell lung cancer: a pooled analysis. Lancet Oncol 2019;20:1395-40.

15. Reck M, Rodriguez-Abreu D, Robinson AG, et al. on behalf of the KEYNOTE-024 investigators. Pembrolizumab versus chemotherapy for PD-L1-positive non-small-cell lung cancer. $N$ Engl J Med 2016;375:1823-33.

16. MokTSK, Wu YL, Kudaba I, et al. on behalf of the KEYNOTE-042 investigators. Pembrolizumab versus chemotherapy for previously untreated, PD-L1-expressing, locally advanced or metastatic non-small-cell lung cancer (KEYNOTE-042): a randomised, open-label, controlled, phase 3 trial. Lancet 2019;393:1819-30.

17. Carbone DP, Reck M, Paz-Ares L, et al. on behalf of the CheckMate 026 investigators. First-line nivolumab in stage IV or recurrent non-small-cell lung cancer. $N$ Engl J Med 2017; 376:2415-26.

18. Planchard D, Yokoi T, McCleod MJ, et al. A phase III study of 
durvalumab (MEDI4736) with or without tremelimumab for previously treated patients with advanced NSCLC: rationale and protocol design of the ARCTIC study. Clin Lung Cancer 2016;17:232-6.e1.

19. Gandhi L, Rodriguez-Abreu D, Gadgeel S, et al. Pembrolizumab plus chemotherapy in metastatic non-small-cell lung cancer. N Engl J Med 2018;378:2078-92.

20. Papadimitrakopoulou V, Cobo M, Bordoni R, etal. IMpower132: PFS and safety results with $1 \mathrm{~L}$ atezolizumab + carboplatin/ cisplatin + pemetrexed in stage IV non-squamous NSCLC [abstract OA05.07]. J Thorac Oncol 2018;13(suppl):S332-3.

21. Jotte RM, Cappuzzo F, Vynnychenko I, et al. IMpower131: primary PFS and safety analysis of a randomized phase III study of atezolizumab + carboplatin + paclitaxel or nab-paclitaxel vs. carboplatin + nab-paclitaxel as $1 \mathrm{~L}$ therapy in advanced squamous NSCLC [abstract LBA9000]. J Clin Oncol 2018;36:. [Available online at: https://ascopubs.org/doi/abs/10.1200/ JCO.2018.36.18_suppl.LBA9000; cited 11 January 2020]

22. Paz-Ares L, Luft A, Vicente D, et al. Pembrolizumab plus chemotherapy for squamous non-small-cell lung cancer. $N$ Engl J Med 2018;379:2040-51.

23. Borcoman E, Kanjanapan Y, Champiat S, et al. Novel patterns of response under immunotherapy. Ann Oncol 2019;30: 385-96.

24. Champiat S, Dercle L, Ammari S, et al. Hyperprogressive disease is a new pattern of progression in cancer patients treated by anti-PD-1/PD-L1. Clin Cancer Res 2017;23:1920-8.

25. Ferrara R, Mezquita L, Texier M, et al. Hyperprogressive disease in patients with advanced non-small cell lung cancer treated with PD-1/PD-L1 inhibitors or with single-agent chemotherapy. JAMA Oncol 2018;4:1543-52.

26. Repetto L. Greater risks of chemotherapy toxicity in elderly patients with cancer. J Support Oncol 2003;1(suppl 2):18-24.

27. Tabchi S, Kassouf E, Florescu M, Tehfe M, Blais N. Factors influencing treatment selection and survival in advanced lung cancer. Curr Oncol 2017;24:e115-22.

28. Marur S, Singh H, Mishra-Kalyani P, et al. FDA analyses of survival in older adults with metastatic non-small cell lung cancer in controlled trials of PD-1/PD-L1 blocking antibodies. Semin Oncol 2018;45:220-5.

29 Elkrief A, Richard C, Malo J, et al. Efficacy of immune checkpoint inhibitors in older patients with non-small cell lung cancer: real-world data from multicentric cohorts in Canada and France. J Geriatr Oncol 2020;:[Epub ahead of print].

30. Kudoh S, Takeda K, Nakagawa K, et al. Phase III study of docetaxel compared with vinorelbine in elderly patients with advanced non-small-cell lung cancer: results of the West Japan Thoracic Oncology Group Trial (WJTOG 9904). J Clin Oncol 2006;24:3657-63.

31. Quoix E, Zalcman G, Oster JP, et al. on behalf of the Intergroupe francophone de cancérologie thoracique. Carboplatin and weekly paclitaxel doublet chemotherapy compared with monotherapy in elderly patients with advanced non-smallcell lung cancer: IFCT-0501 randomised, phase 3 trial. Lancet 2011;378:1079-88.

32. Fehrenbacher L, von Pawel J, Park K, et al. Updated efficacy analysis including secondary population results for OAK: a randomized phase III study of atezolizumab versus docetaxel in patients with previously treated advanced non-small cell lung cancer. J Thorac Oncol 2018;13:1156-70.

33. Herbst RS, Baas P, Kim DW, et al. Pembrolizumab versus docetaxel for previously treated, PD-L1-positive, advanced non-small-cell lung cancer (KEYNOTE-010): a randomised controlled trial. Lancet 2016;387:1540-50.

34. Horn L, Spigel DR, Vokes EE, et al. Nivolumab versus docetaxel in previously treated patients with advanced non-small-cell lung cancer: two-year outcomes from two randomized, open- label, phase III trials (CheckMate 017 and CheckMate 057). J Clin Oncol 2017;35:3924-33.

35. Dogan S, Shen R, Ang DC, et al. Molecular epidemiology of EGFR and KRAS mutations in 3,026 lung adenocarcinomas: higher susceptibility of women to smoking-related KRASmutant cancers. Clin Cancer Res 2012;18:6169-77.

36. Rosell R, Carcereny E, Gervais R, et al. on behalf of the Spanish Lung Cancer Group in collaboration with Groupe français de pneumo-cancérologie and the Associazione Italiana Oncologia Toracica. Erlotinib versus standard chemotherapy as first-line treatment for European patients with advanced EGFR mutation-positive non-small-cell lung cancer (EURTAC): a multicentre, open-label, randomised phase 3 trial. Lancet Oncol 2012;13:239-46.

37. Mitsudomi T, Morita S, Yatabe Y, et al. on behalf of the West Japan Oncology Group. Gefitinib versus cisplatin plus docetaxel in patients with non-small-cell lung cancer harbouring mutations of the epidermal growth factor receptor (WJTog3405): an open label, randomised phase 3 trial. Lancet Oncol 2010;11:121-8.

38. Sequist LV, Yang JC, Yamamoto N, et al. Phase III study of afatinib or cisplatin plus pemetrexed in patients with metastatic lung adenocarcinoma with EGFR mutations. J Clin Oncol 2013;31:3327-34.

39. Soria JC, Ohe Y, Vansteenkiste J, et al. on behalf of the FLAURA investigators. Osimertinib in untreated EGFRmutated advanced non-small-cell lung cancer. NEnglJ Med 2018;378:113-25.

40. Mok TS, Wu YL, Ahn MJ, et al. on behalf of the AURA3 investigators. Osimertinib or platinum-pemetrexed in EGFR T790M-positive lung cancer. N Engl J Med 2016;376:629-40.

41. Gainor JF, Dardaei L, Yoda S, et al. Molecular mechanisms of resistance to first- and second-generation ALK inhibitors in ALK-rearranged lung cancer. Cancer Discov 2016;6:1118-33.

42. Shaw AT, Kim DW, Nakagawa K, et al. Crizotinib versus chemotherapy in advanced $A L K$-positive lung cancer. $N$ Engl $J$ Med 2013;368:2385-94.

43. Camidge DR, Kim HR, Ahn MJ, et al. Brigatinib versus crizotinib in $A L K$-positive non-small-cell lung cancer. $N$ Engl $J$ Med 2018;379:2027-39.

44. Peters S, Camidge DR, Shaw AT, et al. Alectinib versus crizotinib in untreated $A L K$-positive non-small-cell lung cancer. N Engl J Med 2017;377:829-38.

45. Mazieres J, Drilon A, Lusque A, et al. Immune checkpoint inhibitors for patients with advanced lung cancer and oncogenic driver alterations: results from the IMMUNOTARGET registry. Ann Oncol 2019;30:1321-8.

46. Reck M, MokTSK, Nishio M, et al. on behalf of the IMpower 150 study group. Atezolizumab plus bevacizumab and chemotherapy in non-small-cell lung cancer (IMpower150): key subgroup analyses of patients with EGFR mutations or baseline liver metastases in a randomised, open-label phase 3 trial. Lancet Respir Med 2019;7:387-401.

47. Temel JS, Greer JA, Muzikansky A, et al. Early palliative care for patients with metastatic non-small-cell lung cancer. N Engl J Med 2010;363:733-42.

48. Sullivan DR, Chan B, Lapidus JA, et al. Association of early palliative care use with survival and place of death among patients with advanced lung cancer receiving care in the Veterans Health Administration. JAMA Oncol 2019;:[Epub ahead of print].

49. Brahmer JR, Lacchetti C, Schneider BJ, et al. on behalf of the National Comprehensive Cancer Network. Management of immune-related adverse events in patients treated with immune checkpoint inhibitor therapy: American Society of ClinicalOncology clinical practice guideline.JClin Oncol2018; 36:1714-68. 
50. Thompson JA. New NCCN guidelines: recognition and management of immunotherapy-related toxicity. J Natl Compr Canc Netw 2018;16:594-6.

51. Lee JS, Ruppin E. Multiomics prediction of response rates to therapies to inhibit programmed cell death 1 and programmed cell death 1 ligand 1.JAMA Oncol 2019;:[Epub ahead of print].

52. Fumet JD, Richard C, Ledys F, et al. Prognostic and predictive role of CD8 and PD-L1 determination in lung tumor tissue of patients under anti-PD-1 therapy. Br J Cancer 2018;119:95060. [Erratum in: Br J Cancer 2019;121:283]

53. Hellmann MD, Paz-Ares L, Bernabe Caro R, et al. Nivolumab plus ipilimumab in advanced non-small-cell lung cancer. $N$ Engl J Med 2019;381:2020-31.

54. Peters S, Cho BC, Reinmuth N, et al. Tumor mutational burden (TMB) as a biomarker of survival in metastatic non-small cell lung cancer (mNSCLC): blood and tissue TMB analysis from MYSTIC, a phase III study of first-line durvalumab \pm tremelimumab vs. chemotherapy [abstract CT074]. Cancer Res 2019;79(suppl):. [Available online at: https://cancerres. aacrjournals.org/content/79/13_Supplement/CT074; cited 11 January 2020]
55. Routy B, Le Chatelier E, Derosa L, et al. Gut microbiome influences efficacy of PD-1-based immunotherapy against epithelial tumors. Science 2018;359:91-7.

56. Elkrief A, El Raichani L, Richard C, et al. Antibiotics are associated with decreased progression-free survival of advanced melanoma patients treated with immune checkpoint inhibitors. Oncoimmunology 2019;8:e1568812.

57. Elkrief A, Derosa L, Kroemer G, Zitvogel L, Routy B. The negative impact of antibiotics on outcomes in cancer patients treated with immunotherapy: a new independent prognostic factor? Ann Oncol 2019;30:1572-9.

58. Arbour KC, Mezquita L, Long N, et al. Impact of baseline steroids on efficacy of programmed cell death-1 and programmed death-ligand 1 blockade in patients with nonsmall-cell lung cancer. J Clin Oncol 2018;36:2872-8.

59. Drilon AE, Subbiah V, Oxnard GR, et al. A phase 1 study of LOXO-292, a potent and highly selective RET inhibitor, in patients with RET-altered cancers [abstract 102].J Clin Oncol 2018;36:. [Available online at: https://ascopubs.org/doi/ abs/10.1200/JCO.2018.36.15_suppl.102; cited 11 January 2020]

60. Mattox AK, Bettegowda C, Zhou S, et al. Applications of liquid biopsies for cancer. Sci Transl Med 2019;11:eaay1984. 